\title{
Correlação entre Propriedades Mecânicas e Parâmetros Estruturais de Poliuretanos à Base de Poli(e-caprolactona)
}

\author{
Juliana Kloss, Caroline Bugay, Leni Akcelrud, Sônia F. Zawadzki \\ Departamento de Química, UFPR \\ Shu-Hui Wang \\ Escola Politécnica, USP
}

\begin{abstract}
Resumo: No presente trabalho foram preparados poliuretanos (PUs) segmentados. Primeiramente foi obtido um prépolímero (PP) a partir da reação de 2,4 e 2,6 - diisocianato de tolileno (TDI) e poli(e-caprolactona) diol (PCL). A PCL é um poliéster biodegradável que constituiu o segmento flexível do PU. O segmento rígido foi constituído por unidades uretânicas provenientes da ligação entre as extremidades isocianato do PP e as hidroxilas do extensor de cadeia: 1,4 butanodiol (BDO), ou sacarose (SAC), ou glicose (GLY). Foram avaliadas as propriedades mecânicas e dinâmicomecânicas dos poliuretanos obtidos e estas foram correlacionadas com os parâmetros estruturais. Os resultados foram justificados com base na intensidade das interações de hidrogênio, na mistura de fases, no volume dos extensores cíclicos e na presença de ligações cruzadas. Estes PUs estão sendo estudados com vistas à preparação de materiais biodegradáveis com propriedades mecânicas úteis.
\end{abstract}

Palavras-chave: Poliuretano, poli(E-caprolactona), biodegradação, propriedades mecânicas.

\section{Relationships between Mechanical Properties and Structural Parameters of Polyurethanes Containing Poly( $\varepsilon$-caprolactone)}

Abstract: Segmented polyurethanes containing polycaprolactone as soft segment were prepared taking into account the biodegradable character of this compound. The hard block was produced by the reaction between tolylene diisocyanate and a chain extender (1,4 butanediol, sucrose or glucose). The mechanical and dynamical properties were evaluated as a function of the contents of rigid blocks. The results were analyzed on the basis of the intermolecular interactions, mainly $\mathrm{H}$ bonds, phase mixture, volume of cyclic chain extenders and crosslinking. These polyurethanes are being studied aiming at the preparation of biodegradable materials with useful mechanical properties.

Keywords: Polyurethanes, polycaprolactone, biodegradable materials, mechanical properties.

\section{Introdução}

Diversas alternativas têm sido buscadas para minimizar o impacto ambiental causado pelos polímeros convencionais. Uma delas é o uso de polímeros biodegradáveis, que podem ser degradados pela ação de microrganismos, tais como bactérias, fungos ou algas. Estes materiais são relativamente novos e começaram a surgir na década de $60^{[1-3]}$. As aplicações tecnológicas de polímeros biodegradáveis normalmente requerem melhorias nas suas propriedades mecânicas ou, ao menos, um balanço entre a capacidade de degradação e as propriedades do produto final, além de um custo acessível. Os polímeros biodegradáveis são bastante utilizados na área médica em suturas, implantes, matrizes para liberação controlada de drogas, etc., por serem, muitos deles, biocompatíveis. A utilização em aplicações de maior escala se torna dificultada pelas baixas características de desempenho mecânico e térmico destes materiais. Assim, tornou-se cada vez mais evidente a necessidade de substituir polímeros naturalmente biodegradáveis por polímeros sintéticos, que aliassem o bom desempenho mecânico à biodegradabilidade ${ }^{[4-6]}$.

O desenvolvimento da pesquisa neste campo vem sendo feito através de dois enfoques distintos: no primeiro, são estudadas as possíveis mutações e formações de híbridos de microrganismos conduzindo à formação de novos genótipos (diversidade microbiológica); e no segundo, são testadas estruturas poliméricas no que se refere à biodegradabilidade, com vistas ao estabelecimento de correlações entre características estruturais do polímero e acessibilidade metabólica por parte de microrganismos (síntese de novos materiais $)^{[7-11]}$.

Neste contexto, novos polímeros biodegradáveis, como por exemplo poliuretanos, vêm sendo obtidos para aplicações inovadoras e com menor custo ${ }^{[12-16]}$. Os poliuretanos constituem, atualmente, uma das mais importantes classes 
comercialmente aplicáveis de polímeros. Devido à possibilidade de modificações estruturais, estes possuem ampla aplicação tecnológica em várias áreas tais como: materiais de revestimento, fibras, adesivos, borrachas, espumas e plásticos. $\mathrm{O}$ mercado para produtos à base de poliuretano atingiu, em 2000, um consumo mundial da ordem de 8,5 milhões de toneladas, com previsão de 10,8 milhões em 2004; comprovando ser um dos produtos mais versáteis empregados pela indústria. Atualmente, os poliuretanos ocupam a $6^{\mathrm{a}}$ posição, com cerca de $5 \%$ do mercado dos plásticos mais vendidos no mundo ${ }^{[7,17-19]}$. No presente trabalho, foram preparados poliuretanos (PUs) segmentados utilizando poli( $\varepsilon$-caprolactona) diol $(\mathrm{PCL})^{[14,19,20-22]}$, um poliéster biodegradável ${ }^{[23-29]}$, de massa molar numérica média $(\overline{\mathrm{Mn}}=2000 \mathrm{~g} / \mathrm{mol})$ que constitui o segmento flexível; e 2,4 e 2,6 - diisocianato de tolileno, TDI. Como extensores de cadeia foram utilizados o 1,4-butanodiol (BDO), ou a sacarose (SAC), ou a glicose (GLY). O segmento rígido foi, portanto, constituído por unidades uretânicas provenientes da ligação entre o diisocianato e o extensor. Foram avaliadas as propriedades mecânicas e dinâmico-mecânicas dos PUs obtidos e estas foram correlacionadas com os parâmetros estruturais. Estes PUs estão sendo estudados com vistas à preparação de materiais biodegradáveis com propriedades mecânicas úteis ${ }^{[3,15]}$.

\section{Experimental}

\section{Materiais}

Poli(E-caprolactona) diol (PCL): Akros $\overline{\mathrm{M}}_{\mathrm{n}}=2000 \mathrm{~g} / \mathrm{mol}$; seca em evaporador rotatório a $80^{\circ} \mathrm{C}$ e $10 \mathrm{mmHg}$ por 3 horas.

2,4 e 2,6 - Diisocianato de tolileno (TDI), mistura 80/20: Hilax; destilado em grade de destilação 14/20 à pressão reduzida de $10 \mathrm{mmHg}$ à temperatura de aproximadamente $123{ }^{\circ} \mathrm{C}^{[30,31]}$.

1,4-Butanodiol (BDO): seco sob constante agitação magnética com hidreto de cálcio durante 48 horas e, em seguida, destilado em grade de destilação 14/20 à pressão reduzida de $10 \mathrm{mmHg}$, à temperatura de $120^{\circ} \mathrm{C}$. O destilado foi redestilado sob as mesmas condições ${ }^{[30,32]}$.

Glicose (GLY): Biotec, seca em pistola de secagem com pentóxido de fósforo, sob vácuo, por 48 horas ${ }^{[32,33]}$.

Sacarose (SAC): Reagen, o reagente foi macerado e seco em pistola de secagem com pentóxido de fósforo, sob vácuo, por 48 horas $^{[32]}$.

\section{Obtenção e caracterização dos poliuretanos}

Obtenção do pré-polímero (PP): em um balão de vidro foram colocados quantidades estequiométricas de PCL-2000 e TDI. Este último foi adicionado em excesso para garantir que toda PCL fique com extremidades isocianato (-NCO), caracterizando a porcentagem de NCO livre (1, 3, 5 e 7\%), garantindo a reação posterior com extensor de cadeia (BDO, SAC ou GLY). A razão NCO/OH no PP foi mantida constante e igual a 1,1 .

O balão com a mistura foi aquecido, sob agitação magnética, ao qual foi adaptado um tubo contendo cloreto de cálcio para evitar a entrada de umidade. A temperatura da reação ficou em torno de $60{ }^{\circ} \mathrm{C}$ por 1 hora e 30 minutos. Para finalizar, foi feito vácuo por aproximadamente 25 minutos com aumento gradativo da temperatura.

Obtenção do poliuretano (PU): foi adicionado ao PP, em quantidades estequiométricas, o extensor de cadeia: butanodiol (BDO) ou sacarose (SAC) ou glicose (GLY). A reação foi conduzida a $90{ }^{\circ} \mathrm{C}$ durante 15 minutos. A seguir, foi feito vácuo por 10 minutos e o PU foi transferido para um molde de alumínio redondo (diâmetro de aproximadamente $20 \mathrm{~mm}$ e espessura de $3 \mathrm{~mm}$ ) e também, para um molde no formato da norma ASTM D $638^{[34]}$ para o teste de tração, os quais foram colocados na estufa por 48 horas, na temperatura de $100^{\circ} \mathrm{C}$.

Caracterização estrutural: utilizando o espectrofotômetro de infravermelho, Bomem MB-100, foi realizada a caracterização estrutural parcial dos PUs. Os espectros foram obtidos com uma resolução de $4 \mathrm{~cm}^{-1}$, 20 scans e número de onda de 4000 a $400 \mathrm{~cm}^{-1}$, através da técnica de refletância total atenuada (ATR).

Caracterização mecânica: os ensaios de tração foram realizados de acordo com a Norma ASTM D 638 ${ }^{[34]}$, em uma Máquina de Tração 4467 INSTRON - PAT: 158466, mediante as seguintes condições: temperatura de $25^{\circ} \mathrm{C}$; umidade de $50 \%$; velocidade de alongamento de $50 \mathrm{~mm} / \mathrm{min}$ e uma célula de carga com $100 \mathrm{kgf}$. Foram realizados cinco ensaios para cada amostra, apenas para as reações com 1 e $5 \%$ de NCO livre. A dureza foi avaliada em um Durômetro Shore-A modelo $720 \mathrm{~b}-\mathrm{SB}$, em dupla camada do corpo de prova. O acondicionamento das amostras foi feito à temperatura e umidade relativa do ar em condições ambientes. Foram obtidos e testados 6 corpos de prova para cada PU ( com 1 e $5 \%$ de NCO) sintetizado.

Análise do comportamento termomecânico (DMTA): As amostras foram submetidas à deformação tensional, com deformação máxima de $0,1 \%$. As amostras de PU foram resfriadas até $-100{ }^{\circ} \mathrm{C}$ com auxílio de nitrogênio líquido e, então, aquecidas até $60{ }^{\circ} \mathrm{C}$, a uma taxa de aquecimento de $2{ }^{\circ} \mathrm{C} / \mathrm{min}$. e uma freqüência de $1 \mathrm{~Hz}$, aparelho Reometric, modelo DMTA V.

\section{Resultados e Discussão}

As estruturas químicas dos poliuretanos sintetizados encontram-se descritas na Figura 1. A utilização do BDO como extensor conduziu à formação de um poliuretano linear, enquanto que, utilizando SAC ou GLY foram obtidos poliuretanos reticulados. A caracterização estrutural feita por espectroscopia na região do infravermelho (Figura 2) revelou, em todos os PUs, a ausência de bandas em $3500 \mathrm{~cm}^{-1}$ característico de $v(\mathrm{OH})$ e formação $v(\mathrm{NH})$ em $3300 \mathrm{~cm}^{-1}$, além do $v(\mathrm{C}=\mathrm{O})$ em $1750 \mathrm{~cm}^{-1}$ e $v(\mathrm{COC}) \mathrm{em} 1250 \mathrm{~cm}^{-1}$, indicando a formação do polímero, conforme esperado. Os dados de composição são mostrados na Tabela 1, bem como os valores das temperaturas de transição vítrea ( $\mathrm{Tg}$ ) referentes aos máximos das curvas de $\tan \delta$, obtidas através do DMTA. Avaliando o 
a)

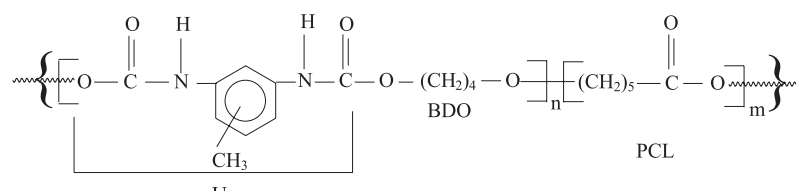

b)

c)

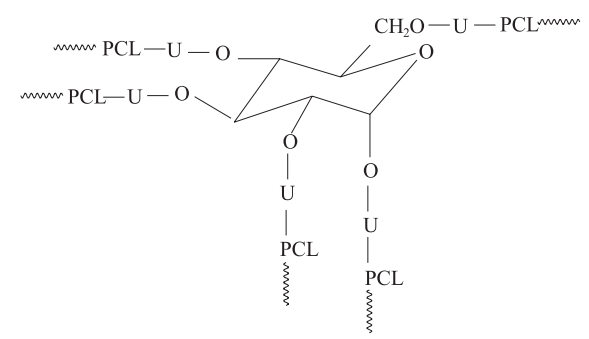

$$
\mathrm{CH}_{2} \mathrm{O}-\mathrm{U}-\mathrm{PCL} \text { mm }
$$
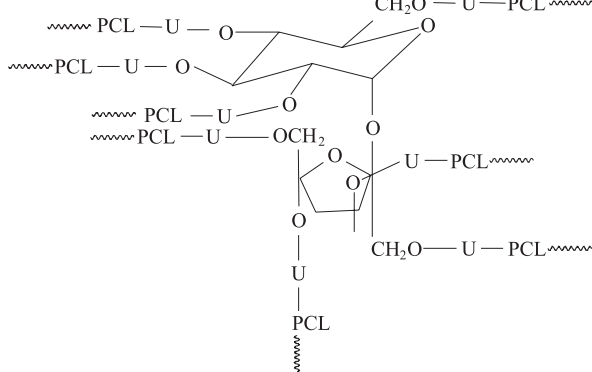

Figura 1. Estruturas dos poliuretanos. a) Poliuretano à base de 1,4butanodiol; b) Poliuretano à base de glicose e c) Poliuretano à base de sacarose.

Tabela 1. Composição dos poliuretanos e seus respectivos valores de $\mathrm{Tg}$

\begin{tabular}{cccc}
\hline PUs/EXT* & $\begin{array}{c}\text { Razão molar } \\
\text { PCL/TDI/EXT }\end{array}$ & $\begin{array}{c}\mathbf{X} \\
(\%) * *\end{array}$ & $\begin{array}{c}\text { Tg } \\
\left({ }^{\circ} \mathbf{C}\right)\end{array}$ \\
\hline 1 BDO & $4 / 5 / 1$ & 11 & $-35,8$ \\
$2 \mathrm{BDO}$ & $1,5 / 2,5 / 1$ & 16 & $-30,6$ \\
$3 \mathrm{BDO}$ & $1 / 2 / 1$ & 21 & $-14,2$ \\
4 BDO & $1 / 3 / 2$ & 26 & $-12,5$ \\
$5 \mathrm{SAC}$ & $18 / 19 / 1$ & 11 & $-36,2$ \\
$6 \mathrm{SAC}$ & $5 / 10 / 1$ & 16 & $-34,1$ \\
$7 \mathrm{SAC}$ & $3 / 7 / 1$ & 21 & $-27,4$ \\
8 SAC & $2 / 6 / 1$ & 26 & $-24,9$ \\
$9 \mathrm{GLY}$ & $12 / 12,5 / 1$ & 11 & $-32,0$ \\
$10 \mathrm{GLY}$ & $2 / 5 / 1$ & 16 & $-31,9$ \\
$11 \mathrm{GLY}$ & $2 / 4 / 1$ & 21 & $-28,6$ \\
$12 \mathrm{GLY}$ & $1,5 / 3,5 / 1$ & 26 & $-24,8$ \\
\hline
\end{tabular}

* Razão $\mathrm{NCO} / \mathrm{OH}=1,1$

${ }^{* *} \mathrm{X}=\left(\mathrm{m}_{\mathrm{TDI}}+\mathrm{m}_{\mathrm{EXT}}\right) /\left(\mathrm{m}_{\mathrm{PCL}}+\mathrm{m}_{\mathrm{TDI}}+\mathrm{m}_{\mathrm{EXT}}\right)$

comportamento térmico, pôde ser observado que, o aumento do teor de segmento rígido $(\mathrm{X})$, acarretou aumento nos valores da $\mathrm{Tg}$, para todas as séries. Este efeito pode ser justificado pelo aumento nas interações hidrogênio devido ao aumento de X. Por outro lado, comparando as séries entre si, pôde-se observar que o efeito foi mais pronunciado para a série linear (com o BDO), este fato pôde ser atribuído ao maior
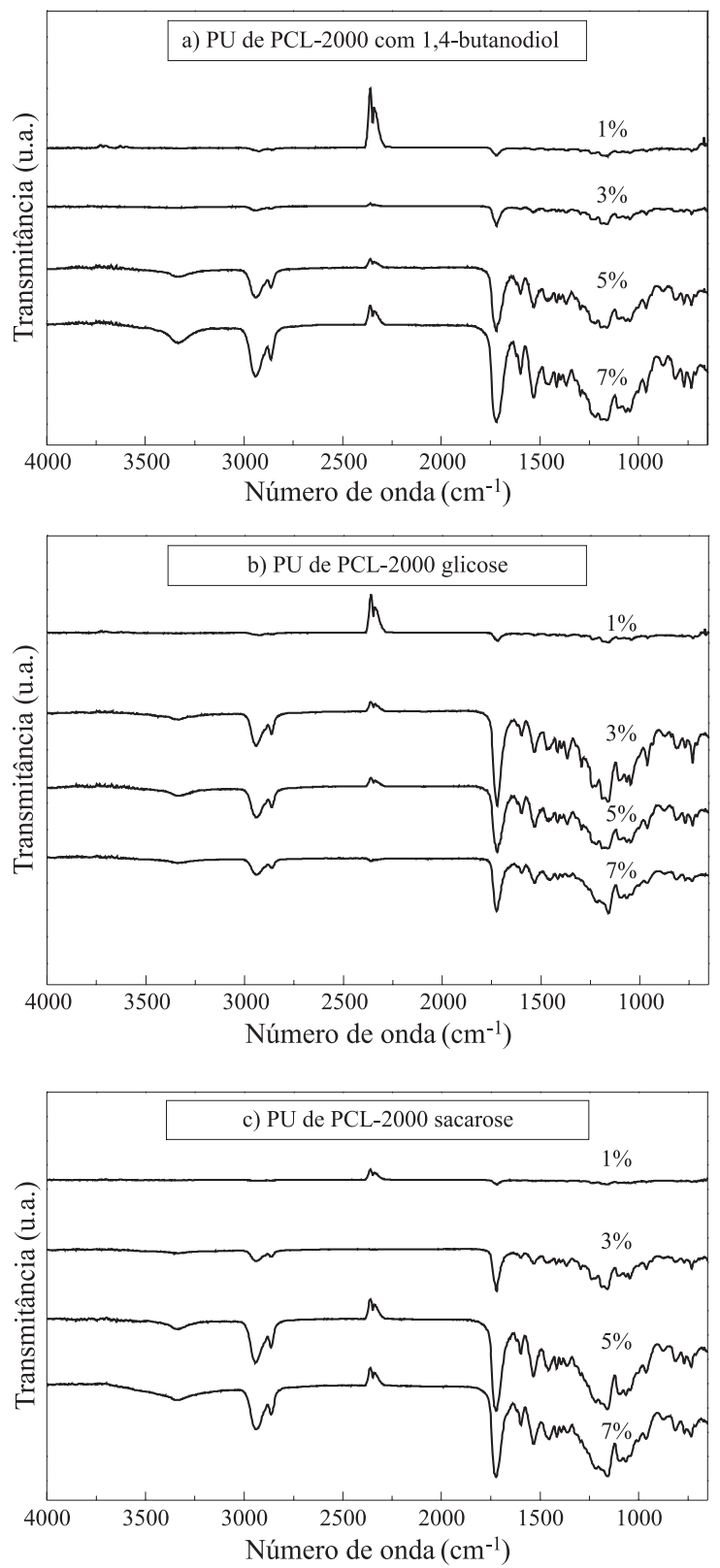

Figura 2. Espectros de infravermelho dos poliuretanos com diferentes extensores e teores de NCO livre iguais a 1,3,5 e 7\%. a) 1,4-butanodiol; b) glicose e c) sacarose.

empacotamento das cadeias lineares em relação às reticuladas com extensores cíclicos, os quais introduzem maior volume livre.

Os resultados relativos ao desempenho mecânico (resistência à tração, módulo de elasticidade, alongamento e dureza) variaram conforme a composição dos polímeros, como mostrado na Tabela 2. Para o extensor alifático, foi observado um aumento na resistência à tração, no módulo de elasticidade e na dureza, com o aumento do teor em segmento rígido (X), o que se justifica pelo aumento do número de ligações de hidrogênio que acompanha a formação de unidades uretano e aos efeitos de empacotamento molecular, conforme discutido anteriormente, na influência da composição sobre a Tg. Na verdade, a melhora nas propriedades mecânicas 
Tabela 2. Dados das propriedades mecânicas obtidas para os poliuretanos.

\begin{tabular}{cccccc}
\hline PUs/EXT & $\begin{array}{c}\mathbf{X} \\
(\boldsymbol{\%})\end{array}$ & $\begin{array}{c}\text { Alongamento } \\
(\boldsymbol{\%})\end{array}$ & Dureza & $\begin{array}{c}\text { Módulo de elasticidade } \\
(\mathbf{M P a})\end{array}$ & $\begin{array}{c}\text { Resistência à tração } \\
(\mathbf{M P a})\end{array}$ \\
\hline 1 BDO & 11 & $33,67 \pm 1,02$ & $41,6 \pm 1,4$ & $24,40 \pm 3,55$ & $3,13 \pm 1,08$ \\
$3 \mathrm{BDO}$ & 21 & $601,8 \pm 6,06$ & $45,5 \pm 2,0$ & $68,60 \pm 20,31$ & $17,1 \pm 7,14$ \\
$5 \mathrm{SAC}$ & 11 & $484,6 \pm 1,87$ & $41,8 \pm 3,2$ & $221,2 \pm 9,36$ & $16,2 \pm 2,98$ \\
$7 \mathrm{SAC}$ & 21 & $125,7 \pm 12,5$ & $39,6 \pm 3,4$ & $198,0 \pm 16,70$ & $5,89 \pm 0,94$ \\
$9 \mathrm{GLY}$ & 11 & $255,4 \pm 14,4$ & $46,0 \pm 2,5$ & $307,2 \pm 16,70$ & $22,7 \pm 2,13$ \\
$11 \mathrm{GLY}$ & 21 & $212,1 \pm 16,6$ & $41,0 \pm 1,0$ & $275,4 \pm 17,3$ & $8,60 \pm 1,66$ \\
\hline
\end{tabular}

e o aumento da transição vítrea, são expressões da coesão intermolecular e deslocam-se no mesmo sentido. No entanto, para os extensores cíclicos e polifuncionais, SAC ou GLY, o efeito oposto foi observado: as propriedades mecânicas diminuíram com aumento do teor em segmento rígido $(\mathrm{X})$. $\mathrm{Na}$ propriedade alongamento na ruptura, foi observado um comportamento diferenciado: para o BDO, a propriedade aumentou com o teor de $(\mathrm{X})$, isto pode ser justificado pela mistura de fases, segmento rígido com flexível. Para a GLY e para a SAC, o alongamento diminuiu nos poliuretanos, devido, provavelmente, à presença de ligações cruzadas. Os resultados apresentados indicam que as forças interativas introduzidas pelas ligações hidrogênio são mais importantes na coesão intermolecular do que as ligações cruzadas. Este efeito foi encontrado anteriormente em poliuretanos preparados a partir de extensores alifáticos e aromáticos ${ }^{[35]}$.

\section{Conclusões}

Foram sintetizadas três séries de poliuretanos segmentados tendo policaprolactona, polímero biodegradável, como bloco flexível. As propriedades térmicas e o desempenho mecânico apresentaram-se como a resultante de um balanço entre empacotamento molecular, teor em ligações hidrogênio, presença de ligações cruzadas e, possivelmente, mistura de fases.

$\mathrm{Na}$ análise das propriedades mecânicas verificou-se que a série de melhor desempenho foi: PCL + TDI + GLY $(X=11 \%)$, dependendo da aplicação a que este material seja submetido.

\section{Agradecimentos}

CNPq, LABPOL; DQ-UFPR; LACTEC; FAPESP (99/ 01783-0).

\section{Referências Bibliográficas}

1. Rosa, D.dos S.; Franco, B. L. M.\& Calil, M. R. - Polím. Ciên. e Tecnol., 11(2), p.82 (2001).

2. Rosa, D. dos S. - Polím. Ciên. e Tecn., 12(4), p.E16 (2002).
3. Kloss, J. R.; Munaro, M.; Souza, G.P.; Gulmine, J. V.; Wang, S.H.; Silva, L.F.; Zawadzki, S. \& Akcelrud, L. - Polym. Sci., 40, p.4117(2002).

4. Immirzi, B.; Malinconico, M.; Orsello, G.; Portofino, M. \& Volpe, M. G. - J. Mat. Scien., 34, p.1625 (1999).

5. Hsieh, K.H.; Tsai, C.C. \& Tseng, S.M. - J. Membr. Scien.; 49, p.341 (1990).

6. Almeida, C. L.; Gonçalves, D.; Pinto, M. R. \& Akcelrud, L. - Polím. Ciên. e Tecnol, 10(4), p.193 (2000).

7. Kim, Y. D. \& Kim, S.C. - Polym. Degrad. And Stabli., 62, p.343 (1998).

8. Andrady, L. A. - "Physical Properties of Polymers Handbook", J. E. Mark, New York (1996).

9. Alexander, M. - Adv. in Appl. Microb., 7, p.35 (1965).

10. Huang, S. J. - "Encyclopedia of Polymer Science \& Engineering”, John Wiley of Sons, New York (1985).

11. Burge, W. D.- Appl. Microb., 17, p.545 (1969).

12. Howard, G. T. - Intern. Biodet. \& Biodeg., 49. p.245 (2002).

13. Phakadze, G.; Grigorieva, M.; Gladir, I \& Momot, V. - J. of Mat. Scien.: Mat. in Med.. 7, p.265 (1996).

14. Groot, J. H.; Vrijer, R. de; Wildeboer, B. S.; Spaana, C. S. \& Pennings, A. J. - Polym. Bull, 38, p.211 (1997).

15. Wang, S. H.; Silva, L. F.; Kloss, J.; Munaro, M.; Souza, G. P. de.; Wada, M. A.; Gomez, J. G.; Zawadzki, S. \& Akcelrud, L. - Macromol. Symp.; 197, p.255 (2003).

16. Bogdanov, B.; Toncheva, V. \& Schacht, E. - J. of Therm. Anal. and Calorim., 56, p.1115 (1999).

17. Backus, J. K. - "Polyurethanes of Polymer Science and Engineering", John-Wiley \& Sons Inc., New York (1988).

18. www.polyurethanes.com.br

19. Sánchez-Adsuar, M. S.; Pastor-Blas, M. M.; MartínezMartín, J.M. \& Villenave, J.J. - Int. J. Adhesion and Adhesives., 17(2), p.155 (1997). 
20. Bogdanov, B.; Toncheva, V.; Schach E.; Finelli, L. \& Scandola, M. - Polym, 40, p.3171(1999).

21. Hirt, T. D.; Neuenschwander, P. \& Suter, U. W. - Macromol. Chem. Phys., 197, p.4253 (1996).

22. Kim, B.K.; Lee, S. Y. \& Xu, M. - Polym., 37(26), p.5781 (1996).

23. Goldberg, D. - J. of Enviorm. Polym. Degrad., 3(2), p.61 (1995).

24. Tsuji, H. \& Suzuyoshi, K. - Polym. Degrad. and Stab., 75, p.347 e 357 (2002).

25. Kressler, J. \& Wang, C. - Lang., 13, p.4407 (1997).

26. Kesel, C. De.; Lefévre, C.; Nagy, J. B. \& David, C. Polym., 40(8), p.1969 (1999).

27. Oda, Y.; Asari, H.; Urakami, T. \& Tonomura, K. - J. of Ferm. and Bioeng., 80(3), p.265 (1995).

28. Sanchez, J.G.; Tsuchii, A. \& Tokiwa, Y. - Biotechn. Lett., 22, p.849 (2000).

29. Murphy, C. A.; Cameron, J. A.; Huang, S. J. \& Vinopal, R. T. - App. and Environ. Microb., 62(2), p. 456 (1996).
30. Santos, E. M.; Aguiar, M.; Oliveira-César, M. A. F.; Zawadzki, S. F. \& Akcelrud, L. - Polím. Ciên. e Tecnol., 10(2), p.64 (2000).

31. Stecher, P. G. - "The Merck Index - an Encyclopedia of Chemicals and Drugs", Ed. Merck \& Co. In, Rahway, New Jersey - USA (1968).

32. Perrin, D. D.; Armarego, W. L. F. - "Purification of Laboratory Chemicals", Great Britain: Pergamon Press, (1988).

33. The Merck Index - "An Encyclopedia of Chemicals, Drugs and of Biologicals", Merck e Co. In Whitehouse Station, USA (1996).

34. American Society for Testing and Material - ASTM. Annual Book of ASTM Standart, "Standard method of test for tensile properteis of plastics", ASTM D 638, p.223 (1979).

35. Zawadzki, S. F. \& Akcelrud, L. - Polym. Intern. 42, p.422 (1997).

Enviado: $25 / 11 / 03$

Reenviado: 23/08/04

Aprovado24/09/04 